\title{
MULTICULTURALIDAD E INTERCULTURALIDAD: DESAFÍOS EPISTEMOLÓGICOS DE LA ESCOLARIZACIÓN DESARROLLADA EN CONTEXTOS INDÍGENAS ${ }^{1}$ \\ Multiculturalism and Interculturalism: Epistemological Challenges of Schooling in Indigenous Contexts
}

Daniel Quilaqueo R.* Héctor Torres $C$.**

\section{Resumen}

El artículo aborda los conceptos de multiculturalidad, interculturalidad y educación intercultural, como perspectivas teóricas que permiten explicar la dinámica intercultural de la acción educativa y como desafío epistemológico de los conocimientos indígenas en la escolarización. Para ello se realiza un análisis de los elementos teóricos que sustentan estos conceptos; se problematiza la dificultad epistemológica de la educación intercultural, considerando el contexto en que se lleva a cabo, y en consecuencia, se plantea que la dinámica de estos conceptos permite la posibilidad de considerar la interculturalidad tanto para la formación como para la problematización de situaciones interculturales que están en proceso de investigación.

Palabras clave: Multicultural, intercultural, educación intercultural, desafíos epistemológicos.

Abstract

This article addresses the concepts of multiculturalism, interculturalism and intercultural education as theoretical perspectives to explain the intercultural dynamic of educational activities, and as epistemological challenges to indigenous knowledge in the school context. To examine these concepts we analyze the theoretical foundations which underlie them. We then formulate the epistemological problem of intercultural education, considering the context in which it takes place. Consequently, we argue that it is the dynamic of these concepts which makes it possible to consider interculturalism both in education and in the formulation of the questions posed by the intercultural situations under investigation.

Key words: Multicultural, intercultural, intercultural education, epistemological challenges.

\section{INTRODUCCIÓN}

Se presentan dos perspectivas que permiten explicar la dinámica intercultural de la escolarización en los contextos de vida de los pueblos indígenas de Chile. La primera se refiere a los significados de los conceptos multicultural, intercultural y educación intercultural asumidos por el medio académico nacional e internacional

\footnotetext{
${ }^{1}$ Este artículo es parte de los resultados del Proyecto Fondecyt № 1110677 y de los Talleres Metodológicos del Núcleo Milenio, Centro de Investigación en Educación en Contexto Indígena e Intercultural CIECII.
} 
desde un punto de vista tanto epistemológico como histórico. La segunda perspectiva plantea la dificultad epistemológica que implica la formación a la interculturalidad en el medio escolar entre el contexto de descubrimiento y el contexto de justificación de los saberes y conocimientos (Muñoz y Velarde, 2000). La diversidad y la complejidad de representaciones de lo intercultural, en una época donde los cambios profesionales son frecuentes, obligan a los investigadores a considerar la extensión, diversidad y profundidad de los problemas que presentan los contextos interculturales. Como conclusión, se plantea que la dinámica del concepto permite considerar la interculturalidad tanto para formarse como para problematizar situaciones interculturales en investigación.

\section{MULTICULTURALIDAD E INTERCULTURALIDAD}

Los términos multicultural e intercultural no son expresiones intercambiables. Ambos se deben considerar como referencias para comprender y explicar la interculturalidad en los contextos de vida de los pueblos indígenas, principalmente desde la educación propia y la educación escolar (Quilaqueo y Quintriqueo, 2008; Quilaqueo, 2012). Por su parte, Catherine Walsh (2005) se refiere al concepto multicultural en su propuesta epistémica de interculturalidad, donde señala que se caracteriza por los siguientes aspectos: 1) tener un origen conceptual en los países occidentales; 2) adquirir un carácter descriptivo que destaca la presencia de diferentes culturas; 3) evitar las relaciones entre las múltiples culturas presentes en un territorio; 4) fundamentarse en la estructura y principios del Estado liberal; 5) centrarse en la tolerancia del otro, pero como una forma de evitar los conflictos, y 6) ocultar las desigualdades sociales y mantener intactas las estructuras e instituciones de la sociedad.

Considerando lo anterior, en este trabajo, lo multicultural significa la existencia de varias culturas presentes en un mismo territorio y es lo que permite explicar el desafío que presenta la incorporación de los conocimientos indígenas en la escolarización de estudiantes de origen tanto indígena como no indígena. Así, la existencia de varias culturas dentro de un mismo territorio puede darse en una región determinada, en un país o en un continente y sus límites territoriales adquieren diversas formas y obedecen a diversas razones. Desde una perspectiva mundial, la separación de las zonas culturales o de civilizaciones diferentes es de tipo geohistórico (Demorgon, 1998). Esta multiculturalidad de civilización, con sus orientaciones culturales históricas, es evidente en todos los continentes, aunque las grandes zonas culturales diferentes, particularmente las religiosas, han sido regiones que están aisladas unas de otras (Breton, 1987; Todorov, 1993; Wieviorka, 1996).

Históricamente, según Demorgon (1998), son cuatro los tipos de sociedades que forman parte de las características multiculturales del planeta. ${ }^{2}$ Sin embargo, en la

\footnotetext{
${ }^{2}$ Las grandes orientaciones culturales históricas son las sociedades comunitarias y la realeza (en el caso de Europa) o el imperio (en el caso de China), que durante mucho tiempo lucharon entre ellas. De las luchas y alianzas, surge una tercera forma de organización, altamente diversificada, es el caso de las naciones 
mayoría de los pueblos indígenas, de los países latinoamericanos, se identifican principalmente sociedades comunitarias vinculadas al comercio y la sociedad de la información. Donde la población y sus comunidades sufren carencias económicas y han sido socialmente discriminados (Van Dijk, 2007). Así, la noción de multiculturalidad representa las realidades geohistóricas, políticas, sociales y culturales de las sociedades indígenas de América Latina, pero al mismo tiempo es evidente que las principales formas culturales de las comunidades indígenas se han desarrollado a partir de los intercambios singulares con las sociedades dominantes de los estados nacionales. Se trata de intercambios que fueron producidos por relaciones generalmente violentas (Quilaqueo, 2009). Además, la transición entre las formas culturales y sociales, la violencia y también los acuerdos entre ambos tipos de sociedades revelaron la importancia de los intercambios comerciales y evidenciaron el aspecto intercultural de las relaciones. Esto se demuestra, por ejemplo, en los encuentros y contactos entre los mapuches con comerciantes y misioneros (Coña, 1984; Bengoa, 1996; Orellana, 2005).

Ahora bien, cuando las relaciones han estado basadas en la dominación de los pueblos indígenas como la radicación en reducciones, ${ }^{3}$ en el caso mapuche (Bengoa, 2004), se observa que la situación multicultural se hace evidente en el distanciamiento y el aislamiento de las sociedades y los grupos humanos (Wieviorka, 1996). En efecto más allá de los momentos de contacto y de experiencia religiosa, política, económica, por ejemplo, y más allá de las guerras desgarradoras, muchas veces, los aspectos de sobrevivencia buscados por las comunidades indígenas con las sociedades dominantes del Estado-Nación han impuesto acuerdos que conllevan nuevas condiciones de separación en los territorios que fueron ocupados por los indígenas (Cantoni, 1978; Orellana, 1994). Este es el caso de la mayoría de los pueblos indígenas de los países latinoamericanos, luego de la formación de las repúblicas independientes de España y Portugal, donde se observa un proceso de incorporación del indígena a las normas de los Estados, caracterizado por sustentarse en una política colonial de los independentistas criollos de origen europeo basada en la marginación, el racismo y la discriminación social de los pueblos indígenas (Bartolomé, 2010). Desde entonces, con la conquista de los territorios ocupados por los indígenas, por parte de los colonizadores, se crearon zonas de refugios o de reducciones (Aguirre Beltrán, 1967). Allí, los Estados establecieron relaciones interétnicas con las comunidades indígenas, donde se crearon

comerciantes. Sin embargo, a fines del siglo XX surgen las condiciones de una cuarta forma de sociedad, reconocida como sociedad de la información, que se extiende rápidamente por el mundo. Por otra parte, "en numerosas regiones del mundo se encuentran todavía sociedades comunitarias. Estas han subsistido por razones geográficas ligadas a condiciones biológicas extremas" (Demorgon, 1998:30).

${ }^{3}$ En el caso mapuche, la reducción se refiere al tipo de tenencia de tierra que el Estado chileno creó para reagrupar a los mapuches sobrevivientes de la guerra denominada "Pacificación de La Araucanía". Hoy, una parte de la población mapuche habita en contextos rurales y ocupa lotes de tierra en nuevas comunidades, como resultado de la organización sociopolítica creada por el Estado (Ley indígena $\mathrm{N}^{\mathrm{o}}$ 19.253). Pero la mayoría de la población mapuche habita hoy en contextos urbanos (INE, 2008). 
condiciones multiculturales (Marroquin, 1977), es decir, contextos socioculturales que han favorecido la dominación colonial y racial impuesta en Latinoamérica. Estas relaciones se establecieron mediante un tipo de pensamiento particular que ha definido la estructuración de los sistemas de poder y saberes coloniales (Quijano, 2000).

La escuela, por su parte, ha funcionado sistemáticamente para reproducir y perpetuar el pensamiento eurocéntrico (Walsh, 2007), lo que ha generado una relación de dominación y discriminación sociocultural hacia los estudiantes de origen indígena desde una escolarización monocultural (Quilaqueo et al., 2007). Este tipo de escolarización se caracteriza porque los conocimientos socioculturales indígenas, particularmente los saberes educativos, han sido excluidos del currículum escolar tanto para los no indígenas como para los indígenas. Es decir, se ha construido un escenario socioeducativo en donde no se ha reconocido la multiculturalidad como producto de las relaciones interétnicas entre el Estado y las comunidades indígenas (Quilaqueo, 2005).

\section{MULTICULTURALISMO Y EDUCACIÓN MULTICULTURAL}

En países de América del Norte, como Canadá, se promueve un modelo de ciudadanía multicultural en la Carta Canadiense de Derechos y Libertades de 1982, reafirmada por una ley de 1988 respecto de la valorización del multiculturalismo (Esses y Gardner, 1996). En tanto, en Estados Unidos el concepto multicultural se refiere a los grupos de inmigrados como producto de la política migratoria, que denominan melting pot, es decir, la integración de inmigrantes de diferentes condiciones sociales a una misma cultura (Banks, 1995, 2009; Sleeter y Grant, 1988; Antolinez Domínguez, 2011). Por su parte, en Europa el concepto multicultural se utiliza para referirse especialmente a los inmigrantes que deben integrarse a un estado nacional cuya tradición cultural ha sido construida desde hace varios siglos (Demorgon, 1998; Verma, 1984; García, Pulido y Montes, 1997). En América Latina, por otro lado, el término multicultural se aplica principalmente a los pueblos indígenas, como minorías étnicas, según la formulación política de cada país y la implementación de la Educación Intercultural Bilingüe (Quilaqueo, 2005; Dietz, 2008).

Ante la cuestión de cómo debe adaptarse la escuela a la evolución plural de la población, Abdallah-Pretceille (1999) señala que la corriente multicultural establecida desde el plano social y político está sólida enraizada en el medio escolar. Para esta autora, "la educación multicultural es, después de muchos proyectos educativos, una tentativa de control por parte del Estado de la dinámica cultural y adaptación de los sistemas de enseñanza a las necesidades de los diferentes grupos culturales" (1999:28). ${ }^{4}$

Se observa que la situación multicultural actual de los pueblos indígenas ha cambiado a partir de la disminución demográfica de sus comunidades de origen y el

\footnotetext{
${ }^{4}$ En relación con esta idea, en varias universidades de los Estados Unidos se entrega una formación ligada a estudios étnicos y educación multicultural. Así, la educación multicultural se dirige esencialmente a las minorías étnicas o a los emigrantes. Algunos programas de estudios elaboran currículum de carácter multicultural que tienen en cuenta los grupos de emigrantes como los hispánicos o las primeras naciones (Banks, 1997).
} 
aumento de ella en el medio urbano, como resultado de las migraciones sucesivas y finalmente su traslado definitivo a las principales ciudades (Gundermann y González, 2008). Esta situación ha dado paso a numerosas proximidades espaciales y entornos culturales parcialmente comunes, por ejemplo, en el caso de Chile, la cohabitación en una misma zona geográfica, en contextos urbanos y en actividades generales como la política y el comercio. En síntesis, las proximidades espaciales y los entornos culturales han generado términos y conceptos que ilustran, de alguna manera, la percepción de la sociedad multicultural y del derecho de las minorías. A la luz de esta situación, las instituciones públicas y privadas manifiestan su voluntad de promover un nuevo trato con las poblaciones indígenas discriminadas y que, además, son las más desfavorecidas económicamente (Schmelkes, 2010). Esto es, por lo menos, lo que se observa en la agenda política y en las leyes de discriminación positiva o de la acción afirmativa de los Estados (Costa-Lascoux, 1999).

En el contexto actual de los pueblos indígenas, las relaciones que mantienen con los estados nacionales son generalmente de tipo multicultural. Son vistos económicamente como pobres, socialmente inferiores, pero con una gran riqueza cultural y lingüística (Merino, Pilleux, Quilaqueo y San Martín, 2009); Quilaqueo, Quintriqueo y Llanquinao, 2012). Así, la pobreza material es vista como símbolo del ser indígena sin considerar sus patrimonios culturales. En este sentido, se puede constatar que "la investigación social cualitativa y los mismos datos estadísticos estatales revelan que los pueblos originarios siguen siendo víctimas de la explotación económica, la inferiorización y exclusión social y la discriminación ideológica" (Bartolomé, 2010:15). De esta forma, se mantiene una representación desde el marco socioeconómico que inferioriza, limita y excluye la matriz cultural indígena como una alternativa para reconfigurar el desarrollo social tanto local como global (Quilaqueo, 2012). En el caso mapuche, ${ }^{5}$ los textos jurídicos dan cuenta de que las relaciones entre las comunidades y el Estado han sido relaciones que, desde los años 80 del siglo pasado, incluyen los conceptos multicultural e intercultural para referirse a las relaciones establecidas en el proceso de democratización del país, respecto del tipo de educación para los indígenas.

Desde otra perspectiva utilizada en la mayoría de los países de América Latina, los términos multicultural e intercultural se refieren a la educación intercultural bilingüe. Esta educación es sugerida por dirigentes indígenas e intelectuales de las ciencias sociales, con el objeto de contextualizar los saberes escolares con los saberes culturales y educativos de los pueblos indígenas (Quilaqueo, Quintriqueo y Cárdenas, 2005;

\footnotetext{
${ }^{5}$ En efecto, los mapuches constituyen uno de los pueblos indígenas más numerosos del país y han habitado sucesivamente en cuatro tipos de contextos socioculturales: 1) en espacios territoriales según su propia orientación sociopolítica y cultural; 2) en reducciones, que es donde el Estado chileno reagrupó a los sobrevivientes de la guerra llamada "Pacificación de La Araucanía"; 3) en nuevas comunidades, como consecuencia de adjudicaciones de tierras de acuerdo con la Ley Indígena de Protección, Fomento y Desarrollo de los Indígenas de Chile y 4) en el medio urbano, como efecto de la carencia económica por falta de tierras y la sobrepoblación de sus comunidades de origen.
} 
Walsh, 2008). No obstante, para Walsh (2008), Gasché (2010), Dietz y Mateos (2011) y Quilaqueo (2012) el concepto intercultural también se refiere a una relación que compromete saberes culturales y educativos en las relaciones intergrupales y entre personas. Sin embargo, en la perspectiva de las instituciones gubernamentales subyace el punto de vista monocultural y monolingüe, donde lo intercultural es propuesto principalmente para los indígenas (Briones et al., 2006; Quilaqueo et al., 2005; Gasché, 2008). Por su parte, Ferrão identifica la configuración de una interculturalidad dirigida a los grupos minorizados que, desde una perspectiva funcional, “... deben ser integrados a la sociedad nacional” (Ferrão, 2010:339). En esta comprensión del concepto intercultural aflora la idea del multiculturalismo practicado en países donde a las comunidades indígenas se les considera como comunidades culturales separadas, no incorporadas al desarrollo ni a la actividad cultural del país. Para Dietz (2008:11), "el auge reciente del discurso intercultural y de la educación intercultural ha conllevado una recepción crítica y una apropiación selectiva de los paradigmas multiculturales de origen anglosajón". Según este autor, la promulgación de políticas públicas de discriminación positiva como mecanismo de antidiscriminación "ilustran la creciente 'migración discursiva' de enfoques político-pedagógicos desde los clásicos países emisores del multiculturalismo (Canadá, Estados Unidos, Reino Unido, Australia) tanto hacia Europa como hacia Latinoamérica" (Dietz, 12).

$\mathrm{Al}$ referirse a la educación en áreas indígenas de América Latina, Inge Sichra (2003) y Luis Enrique López plantean un enfoque lingüístico-cultural, cuyo objetivo es favorecer a los estudiantes de origen indígena en el desarrollo de su lengua vernácula y el aprendizaje del castellano como vehículo de comunicación con la sociedad hegemónica, buscando fortalecer el pluralismo cultural. Estos autores subrayan que "la educación intercultural bilingüe no puede entenderse como un modelo rígido (...) sino como una estrategia educativa que debe ser adecuada y diferenciada en su ejecución a las características sociolingüísticas y socioculturales de los educandos y sus comunidades" (Sichra y López, 2003:22).

Igual que el caso de los mapuches, es importante la participación indígena en las propuestas educativas, especialmente desde el aspecto sociocultural, es decir, la incorporación de sus visiones tradicionales para el diálogo e interacción con la sociedad hegemónica. Por otra parte, como opción política educativa, entre las variadas interpretaciones del término interculturalidad, se puede rescatar que el cambio de las relaciones sociales y culturales desde una perspectiva de equidad y de pertinencia es de relevancia para la innovación curricular y, de esta manera, atender la diversidad sociocultural.

No obstante, en América Latina se observan todavía políticas específicas para los pueblos indígenas. Se trata del legado indigenista o postindigenista que ha influido en la manera como se ha adaptado el multiculturalismo a las diversas realidades latinoamericanas. En este contexto, lo intercultural y la educación intercultural constituyen hoy temas centrales, tanto en la formación profesional y de persona como a nivel de las ciencias humanas. Así, en la última década, los términos 
multicultural e intercultural aparecen como conceptos que permiten fundamentar las propuestas de acción positiva de las universidades situadas en contextos indígenas (Quintriqueo, 2010). Del mismo modo, en varios países se ha propuesto la educación intercultural bilingüe mediante programas para los niveles de educación parvularia, básica y media (MINEDUC, 1990; CONADI, 1993). Al mismo tiempo, la educación intercultural, como tema de investigación, surge principalmente como problema político, sin mayor contenido indígena ni contenido educativo propio desde la visión de los pueblos indígenas. Así, la incorporación de estudiantes a la formación profesional en las universidades emerge como acción de discriminación positiva o, en algunos casos, como reparación histórica (MIDEPLAN, 2009). En tanto, en investigación, la educación intercultural se estudia como diálogo cultural entre indígenas y no indígenas, lo que implica que sea poco crítica respecto de los contenidos desarrollados, como por ejemplo, los estudios sobre contenidos socioculturales y educativos indígenas (Torres y Quilaqueo, 2011).

En el caso de Chile y Argentina, lo intercultural como objeto de estudio es aun reciente (Tamagno, 2009; Quilaqueo y Quintriqueo, 2010), ya que históricamente los jóvenes han quedado rezagados en sus comunidades o han emigrado como mano de obra a los centros urbanos (Gasché, 2008, 2010). Allí, han sido excluidos porque su pertenencia a los Estados nacionales se ha dado mediante una "integración subordinada a un sistema jerárquico y, en el sistema de 'exclusión', fenómeno social y cultural, la pertenencia se da por exclusión; esto es, se pertenece por la forma en que es excluido" (Castro, 2004:135). Entonces, el hecho de ser excluido socialmente se explica señalándose que los no indígenas los han estereotipado y discriminado, situación que los mapuches señalan como discriminación percibida en el contexto laboral y público (Quilaqueo, Merino y Saiz, 2007; Saiz, Merino y Quilaqueo, 2009).

En general, el desarrollo de la educación intercultural bilingüe en América Latina se ha impulsado en países como Guatemala, Ecuador, Perú, Colombia, México, Bolivia y, últimamente, en Chile y Argentina (Chiodi, 1990; Moya, 1996; Quintriqueo, 2009). En estos países, la educación intercultural se relaciona con las demandas de los pueblos indígenas para tener mejores oportunidades y acceso a una educación de mejor calidad. En Chile, se ha fundamentado en el marco de las relaciones de dominación aplicada en el contacto histórico entre mapuches y no mapuches, donde las decisiones sobre las políticas educacionales han tenido un carácter centralizador, primero, por las instituciones coloniales y, después, por las actuales instituciones estatales. En este sentido, el sistema educacional, desde el período colonial hasta hoy, ha privilegiado un sistema cultural dominante, donde las culturas y saberes de los pueblos indígenas han sido excluidos. Del mismo modo, en los países de América Latina esto ha sido un proceso similar en todos los sistemas educacionales, lo que ha generado, en el marco de la formación escolar y las relaciones sociales, una cultura oficial y otras marginales o periféricas (Magendzo, 1986). Así, el carácter histórico, monocultural y centralizado en el cual se creó la educación escolar en Chile, particularmente la Educación Básica, se mantiene hasta hoy, aun cuando 
en las últimas cinco décadas haya surgido una consideración de la diversidad étnica y cultural en el campo de la educación (Magendzo, 2007; Quintriqueo, 2009).

El carácter monocultural de la educación se ha fundamentado en la formación del Estado-Nación chileno en el siglo XIX, donde se enfatizan los principios culturales de civilización, centralismo político y unidad nacional (Egaña, 2000; Magendzo, 2007, Quintriqueo, 2009), lo que se expresa en el reconocimiento de una cultura única, legitimada social e institucionalmente por la escuela (Rokwell, 1995; Perrenoud, 2005; Quintriqueo, 2007). En este contexto, el modelo educativo que se ha configurado tiene como referente el primer mundo (Labarca, 1939) y, en su proceso, no se reconocen las sociedades ni las culturas originarias al interior de la sociedad nacional. Es decir, quedan excluidas otras formas de pensar, conocer y comprender el mundo, lo que condiciona la perspectiva eurocéntrica como el único modelo válido para definir los contenidos educativos (Walsh, 2008). Además, solo a partir de los años noventa del siglo pasado se observa la consideración de la diversidad étnico-cultural indígena en la educación básica, mediante leyes y decretos específicos, sin modificar el sistema en su conjunto. Al respecto, en Chile, la Ley Indígena $\mathrm{N}^{\circ} 19.253$ y la Ley Orgánica Constitucional de Enseñanza No 18.944 (MINEDUC, 1990) y, en el ámbito internacional, el Convenio 169 de la Organización Internacional del Trabajo y la Declaración de la Naciones Unidas sobre los Derechos de los Pueblos Indígenas (Instituto Indigenista Interamericano, 1996) son ejemplos de que hay consideración y un nuevo trato de discriminación positiva hacia los pueblos indígenas del país. En consecuencia, la escuela hoy se presenta como aparato de un Estado moderno, pero que en su esencia continúa siendo monocultural.

$\mathrm{Al}$ respecto, el currículum escolar chileno responde solamente al modelo social y cultural occidental de base eurocéntrica, especialmente el educativo, que ha escolarizado a muchas generaciones de mapuches desde la masificación de la escuela. Este se ha formando desde el imaginario nacional, lo que ha desencadenado la imposición de una relación hegemónica del saber occidental respecto del saber social, cultural y educativo indígena (Quilaqueo, Fernández y Quintriqueo, 2010). De esta manera, se observa en los manuales escolares y recursos educativos no solo estereotipos, sino también el silenciamiento y ocultamiento de los saberes y conocimientos de los pueblos indígenas (Quilaqueo y Merino, 2003; Quintriqueo, Torres, Gutiérrez y Sáez, 2011). Producto de este proceso, la mayor parte de las nuevas generaciones, tanto de jóvenes mapuches como no mapuches, en Chile, desconocen los conocimientos de la sociedad mapuche (Quintriqueo, 2009). Como consecuencia de esta situación, es posible observar que entre niños y jóvenes se niega, se oculta o se asume con dificultad su identidad étnica, lo que revela uno de los aspectos centrales en la transformación de la familia y de la comunidad mapuche actual (Quilaqueo y Quintriqueo, 2008).

\section{DESAFÍO EPISTEMOLÓGICO DE LA EDUCACIÓN INTERCULTURAL}

Desde el punto de vista epistemológico, lo intercultural puede definirse a partir de lo planteado por Kuhn (2002), como un conjunto de proposiciones que forman una 
base a partir de la cual se desarrolla una tradición de investigación. Por su parte, Abdallah-Pretceille (1999) señala que dicha tradición es el primer eje de esta definición y que reposa sobre el reconocimiento de los datos interculturales, como datos construidos y que corresponden a atributos de las personas. Esta autora lo define como discurso, como problemática y como un enfoque, donde lo intercultural depende de un modo de interrogación específica y no de un campo de aplicación particular. Respecto de la idea de método, se advierte que existe el peligro de reducir lo intercultural a una herramienta metodológica, sobre todo, al comparar sin referencias con una filosofía, lo que se define como una experiencia de vida y no como una experiencia doctrinal. Es decir, en términos de Fornet-Betancourt (2001), se busca la transfiguración de lo propio y de lo ajeno sobre la base de lo común, lo que implica la creación de un espacio común determinado por la convivencia.

Desde el punto de vista conceptual y epistemológico, la base fenomenológica de lo intercultural está fundamentada en una filosofía del sujeto, es decir, en una fenomenología que construye el concepto del sujeto como ser libre y responsable en una comunidad de iguales. De esta manera, el enfoque intercultural rompe con el punto de vista objetivista y estructuralista, porque se interesa en la producción de la cultura por el mismo sujeto y en las estrategias desarrolladas sin llegar a postular que el individuo tiene siempre conciencia (Abdallah-Pretceille, 1999). Así, el término intercultural implica una búsqueda de sentido, porque no es solamente un método, es también una ontología, no en el sentido de una teoría a priori, sino de una ontología que se construye por medio de la observación y la interpretación que hacen los sujetos que comparten un espacio sociocultural y geográfico. Entonces, al introducir el punto de vista del sujeto, no se trata de clamar por el resurgimiento de las teorías individualistas respecto de los indígenas, sino de tener en cuenta la red de subjetividades en la cual se encuentra. De lo anterior se desprende que una red de intersubjetividades dentro de una lógica de la diferencia busca cuantificar la alteridad fijándola; en cambio, el discurso intercultural pone su énfasis en las interacciones y recurre a un enfoque comunicacional (Quilaqueo, Quintriqueo y Cárdenas, 2005). En síntesis, el prefijo inter de la palabra intercultural representa la manera cómo vemos al Otro y la manera cómo nos vemos, percepción que no depende de las características del otro o de las mías, sino de las relaciones mantenidas entre el Yo y el Otro.

Ahora bien, desde una perspectiva crítica de la interculturalidad, Jorge Gasché recalca que la dominación-sumisión de los pueblos indígenas se expresa tanto en términos objetivos como subjetivos y señala que "hablar de la interculturalidad como de una relación horizontal, no es más que un eufemismo para disfrazar las relaciones verticales" (Gasché, 2008:273). Esto queda objetivamente de manifiesto por la falta de reconocimiento constitucional como entidades de derecho público y no únicamente como sujetos de interés público. Subjetivamente, las personas indígenas insertas en el medio urbano suelen sentir vergüenza de hablar su lengua indígena y de reconocer prácticas culturales propias (Sartorello, 2009). En este punto, cabe agregar un elemento 
adicional que limita el desarrollo de las personas indígenas y es el que se refiere al enfoque de los programas de educación intercultural que han estado “...destinados a los estratos sociales dominados, en particular los indígenas, con el fin de que ellos 'respeten' y 'toleren' - iA quiénes? - a los que los dominan, los desprecian, los engañan, los explotan” (Gasché, 2010:115). Es decir, el diseño de los programas gira en torno a prácticas focalizadas en los dominados, en lugar de estar centrado en modificar las actitudes y conductas de los dominantes.

En síntesis, la epistemología, entendida como disciplina que tiene como objeto de estudio el conocimiento científico, se refiere a aspectos más específicos sobre los problemas del conocer, del saber y los conceptos relacionados con el sentido y la inteligencia (Castellano e Hinestroza, 2009). Se trata, en definitiva, de una práctica continua y móvil, aunque esté plagada de leyes fijas e inmutables, donde el científicoinvestigador se encarga de desenterrar elementos estáticos que sustentan la ciencia en su generalidad (Jaramillo, 2003). Así, la epistemología va más allá de ser una ciencia utilizada para comprender disciplinas específicas o la historia de la construcción del conocimiento. La epistemología da la posibilidad de estar constantemente descifrando lo que ocurre en el mundo, con el objeto de comprender y confrontar los conocimientos que emergen, porque sabemos que hay paradigmas epistemológicos concurrentes, contradictorios y diferentes (Ardoino, 2005), que permiten generar una epistemología contextualizada e intercultural, porque existen diferentes formas de construir conocimiento en contextos multiculturales y territorios indígenas.

\section{PROBLEMATIZAR LAS SITUACIONES INTERCULTURALES}

Con la finalidad de estudiar las situaciones interculturales, se hace necesario problematizar mediante el planteamiento de hipótesis, supuestos y preguntas, pero, al mismo tiempo, sin olvidar las grandes matrices culturales que, a su vez, son interculturales y que simultáneamente se presentan en función de las situaciones interpersonales e intergrupales. Sin embargo, para identificar las situaciones interculturales, ya sean interpersonales o intergrupales, es necesario tener en cuenta los elementos culturales que se encuentran atados a las múltiples estrategias de las sociedades que se expresan mediante perspectivas políticas, económicas, informativas y religiosas, entre otros. En consecuencia, un enfoque intercultural histórico y estratégico es fundamental para aspirar a una comprensión más integral. De esta manera, tanto los jóvenes universitarios como los investigadores que participan deben tener en cuenta que las situaciones interculturales también los involucran, pero se debe ir más allá de las implicancias inmediatas, es decir, es necesario recurrir a temas que planteen la problematización de la escolarización. ${ }^{6}$

\footnotetext{
${ }^{6}$ Para ello, algunos ejemplos de temas de problematización permiten plantear que el investigador también está involucrado en la interculturalidad, por ejemplo: entre mayoría y minoría en una sociedad nacional; entre el emigrado y la comunidad de acogida; entre cultura nacional (cultura del Estado) y una cultura 
Finalmente, todos estos temas constituyen metatemas que se refieren a lo intercultural desde la perspectiva del campo profesional, pero situar lo intercultural solamente desde la perspectiva de las profesiones lo transforma en un instrumento que se despoja de su complejidad, lo que incidiría en el empobrecimiento de la perspectiva de las prácticas profesionales. Ahora bien, si consideramos que estamos en una época donde los cambios profesionales son frecuentes, cometeríamos un error al no considerar la extensión, la diversidad y la complejidad que implica asumir la interculturalidad como opción de vida y los problemas que se derivan de ello. Es cierto que cada uno lo acotará a su ámbito de trabajo y al nivel socioeconómico al cual pertenece, por esto tenemos presente, en esta exposición, que la diversidad y la complejidad de representaciones de lo intercultural son elementos vitales que se deben considerar para pasar, si es necesario, de un actor a otro, o de un nivel a otro en función tanto de los contextos socioculturales como los de interés profesional y la libertad de cada sujeto.

\section{CONCLUSIONES}

Considerando las dimensiones abordadas en este trabajo, se revela que los desafios epistemológicos de los conocimientos indígenas en el medio escolar se relacionan con la acción y reconocimiento de los estudiantes de origen indígena. Esto requiere de un proceso de configuración de nuevos marcos epistémicos que cambien las estructuras dominantes y coloniales que continúan operando en el medio académico para el desarrollo de la investigación y la implementación de propuestas educativas interculturales. Para ello, un aspecto clave es redefinir el concepto de interculturalidad, que suele quedar estereotipado como interacción de culturas o diálogo para los grupos minorizados, es decir, la interculturalidad implica una orientación ontológica, filosófica y epistemológica basada en la diversidad de las matrices culturales y en la subjetividad e intersubjetividad de las personas involucradas en relaciones que implican consensos y conflictos. Por su parte, desde el ámbito de una interculturalidad crítica, queda abierta la posibilidad de que la interacción entre los sujetos signifique el volver a mirarse para despojarse de prejuicios y estereotipos que pueden ser explícitos o implícitos. En este sentido, una propuesta innovadora en educación intercultural en el contexto regional y latinoamericano requiere de la interpelación de los no indígenas, tanto docentes como estudiantes, para que de esta forma se supere la situación de negación, discriminación y racismo de los grupos minorizados. Desde esta perspectiva, los nuevos marcos legales y el análisis crítico de las corrientes funcionalistas, que han tenido como propósito impulsar la asimilación y homogenización de los pueblos indígenas, constituyen una alternativa que invita a considerar un nuevo paradigma que asuma en la interculturalidad la posibilidad de visibilizar a los indígenas y de transformar las estructuras académicas alienantes. Finalmente, los aspectos que pueden orientar la construcción de relaciones interculturales sustentadas en el conocimiento multicultural e

indígena; entre la cultura regional y mapuche o la cultura de la ecología; entre la cultura nacional o regional y la cultura virtual; entre la cultura de la escuela y la cultura de las comunidades indígenas, etc. 
intercultural que considere los desafios epistemológicos de la escolarización que se lleva a cabo en contextos indígenas son: 1) la incorporación de contenidos educativos y socioculturales indígenas en la escolarización, en contextos urbanos y rurales, para estudiantes de origen indígena y no indígena; 2) la generación de condiciones científicoacadémicas para consolidar un paradigma de investigación intercultural y 3) el fomento de la discusión epistémica con un enfoque intercultural en la formación entregada por las universidades desde la investigación.

$$
\begin{array}{r}
\text { Universidad Católica de Temuco* } \\
\text { Facultad de Educación } \\
\text { Manuel Montt 056, Temuco (Chile) } \\
\text { dquilaq@uct.cl } \\
\text { Universidad Laval** } \\
1030 \text { Avenue des Sciences-Humaines, Québec (Canadá) } \\
\text { hector.torres-cuevas.1@ulaval.ca }
\end{array}
$$

\section{OBRAS CITADAS}

Abdallah-Pretceille, Martine. L'éducation interculturelle. Paris : Presse Universitaire de France, 1999.

Aguirre Beltrán, Gonzalo. Regiones de refugio. El desarrollo de la comunidad y el proceso dominical en mestizo América. México: Instituto Indigenista Interamericano, México, 1967.

Antolinez Domínguez, Inmaculada. "Contextualización del significado de la educación intercultural a través de una mirada comparativa: Estados Unidos, Europa y América Latina", en Papeles del CEIC 2. 2011:1-37.

Ardoino, Jacques. Complejidad y Formación. Pensar la educación desde una mirada epistemológica. Buenos Aires: Novedades Educativas, 2005.

Banks, James. Multicultural Education. Dimensions and paradigms. J. Banks (Ed.). The Routledge International Companion to Multicultural Education. New York and London: Routledge, 2009.

------- Multicultural Education: Historical Development, Dimensions, and Practice. J. Banks (Ed.). Handbook of research on multicultural education. New York, MacMillan, 1995.

Bartolomé, Miguel. "Interculturalidad y territorialidades confrontadas", en RUNA 31:1. 2010:9-29.

Bengoa, José. Historia del Pueblo Mapuche. Santiago de Chile: Ediciones Sur, 1996.

----- La memoria olvidada de los pueblos indígenas. Historia de los pueblos indigenas. Santiago de Chile: Publicaciones del Bicentenario, 2004.

Breton, Roland. Géographie des civilisations. Paris: Presses Universitaires de France, 1987. 
Briones, Claudia; Delrío, Walter; Lanusse, Paula; Lazzari, Axel; Lorenzetti, Mariana; Szulc, Andrea; Vivaldi, Ana. Diversidad cultural e interculturalidad como construcciones sociohistóricas. A. Ameigeiras y E. Jure (Comp.). Diversidad cultural e interculturalidad. Buenos Aires: Prometeo/UNGS, 2006.

Cantoni, Wilson. Relaciones del mapuche con la sociedad nacional chilena. UNESCO (Ed.). Raza y Clase en la Sociedad Postcolonial. Madrid: UNESCO, 1978.

Castellano, Ana; Hinestroza, Jorge. "Una perspectiva epistemológica para el estudio de las formaciones socioculturales". Revista de Ciencias Sociales 15, no. 4. 2009:681-692.

Castro, Milka (Ed.). Los Desafios de la Interculturalidad: Identidad, Politica y Derecho. Santiago de Chile: Programa Internacional de Interculturalidad, Vicerrectoría de Investigación y Desarrollo, Universidad de Chile, 2004.

CONADI (Corporación Nacional de Desarrollo Indígena). Ley Indígena $N^{\circ} 19.253$ D. Of. $5^{\circ} 10^{\circ}$ 1993. Temuco: CONADI, 1993.

Coña, Pascual. Testimonio de un cacique mapuche. Primera Edición Imprenta Cervantes 1930, Segunda edición ICIRA 1973. Santiago de Chile: Pehuén Editores, 1984.

Costa-Lascoux, Jacqueline. Citoyenneté et multiculturalisme. M. Hilly y M. Lefebvre (Eds.). Identité collective et altérité. Paris: L'Harmattan, 1999.

Chiodi, Francesco. La educación indígena en América Latina. México, Guatemala, Ecuador, Perú y Bolivia. Quito: Abya-Yala-Unesco, 1990.

Demorgon, Jacques. L'Histoire Interculturelle des Sociétés. Paris: Anthropos, 1998.

Dietz, Gunther. "Los estudiantes indígenas en las universidades latinoamericanas: ¿Nuevos sujetos, nuevos enfoque", en Cuadernos Interculturales 6:10. 2008:1114.

Dietz, Gunther; Mateos, Laura. Interculturalidad y educación intercultural en México: un análisis de los discursos nacionales en su impacto en los modelos educativos mexicanos. México: Secretaría de Educación Pública, 2011.

Egaña, María. La educación primaria popular en el siglo XIX en Chile: Una práctica de política estatal. Santiago de Chile: LOM Ediciones, 2000.

Esses, Victoria; Gardner, Reynolds. "Multiculturalism in Canada: Context and current status", in Canadian Journal of Behavioural Science 28:3. 1996:145-152.

Ferrão, Vera. "Educación intercultural en América Latina: distintas concepciones y tensiones actuales", en Estudios Pedagógicos 36:2. 2010:333-342.

Fornet-Betancourt, Raúl. Filosofía e interculturalidad en América Latina, Intento de introducción no filosófica. En Heise María (ed.) Interculturalidad. Creación de un concepto y desarrollo de una actitud. Lima: Inversiones Hathuey S.A.C., 2001:63-73. 
García, Francisco; Pulido, Rafael; Montes, Ángel. "La educación multicultural y el concepto de cultura", en Revista Iberoamericana de Educación 13. 1997:223256.

Gasché, Jorge. Niños, maestros, comuneros y escritos antropológicos como fuentes de contenidos indígenas escolares y la actividad como punto de partida de los procesos pedagógicos interculturales: un modelo sintáctico de cultura. M. Bertely, J. Gasché y R. Podestá (Coords.). Educando en la diversidad cultural. Investigaciones y experiencias educativas interculturales bilingües. Quito: Abya Yala, 2008.

------ "De hablar de la educación intercultural a hacerla", en Mundo Amazónico 1. 2010:111-134.

Gundermann, Hans; González, Héctor. Pautas de integración regional, migración, movilidad y redes sociales en los pueblos indígenas de Chile, en Universum 23:1. 2008:82-115.

Hess, Remi; Wulf, Chritoph. Parcours, passages et paradoxes de l'interculturel. Paris: Ed. Anthropos, 1999.

INE (Instituto Nacional de Estadísticas). Enfoque estadístico - Pueblos IndígenasBoletín del Instituto Nacional de Estadísticas. Santiago de Chile: INE, 2008.

Instituto Indigenista Interamericano, "Convenio $\mathrm{N}^{\circ} 169$ sobre Pueblos Indígenas y Tribales en Países Independientes, 1989. Organización Internacional del Trabajo OIT", en América Indigena LVIII:3-4.

Jaramillo, Luis, “¿Qué es epistemología?”, en Cinta de Moebio № 18. 2003:15-22.

Kuhn Thomas. El camino desde la estructura. Barcelona: Ediciones Paidós Ibérica, S. A., 2002.

Labarca, Amanda. Historia de la Enseñanza en Chile. Santiago de Chile: Editorial Universitaria, 1939.

MINEDUC (Ministerio de Educación de Chile). Ley Orgánica Constitucional de Enseñanza. Santiago de Chile: MINEDUC, 1990.

Magendzo, Abraham. Currículum y Cultura en América Latina. Santiago de Chile: Academia de Humanismo Cristiano, 1986.

------ Homogeneidad y heterogeneidad curricular: Un tema vinculado con la centralización y la descentralización del currículum. Documento de trabajo. 16 de enero de 2007.

Marroquín, Alejandro. Balance del Indigenismo. México: Ediciones Especiales: 76, Instituto Indigenista Interamericano, 1977.

Merino, María Eugenia; Quilaqueo, Daniel; Saiz, José. "Una tipología del discurso de discriminación percibida en mapuches de Chile", en Revista Signos 41:67. 2008:279-297.

Merino, María Eugenia; Pilleux; Mauricio; Quilaqueo, Daniel; San Martín, Berta. Discursive Racism in Chile: The Mapuche Case, en Van Dijk Teun (Ed.), 
Racism and Discourse in Latin America. Rowman and Littlefield. 2009:95130.

MIDEPLAN (Ministerio de Planificación). Informe de la Comisión Verdad Histórica y Nuevo Trato con los Pueblos Indígenas. Santiago de Chile: MIDEPLAN, 2009.

Muñoz, Jacobo; Velarde, Julián. Compendio de epistemología. Madrid: Editorial Trotta, 2000.

Orellana, Mario. Prehistoria y etnología de Chile. Santiago de Chile: Universidad de Chile, Bravo y Allende Editores, 1994.

Perrenoud, Phillipe. L'école face à la diversité des cultures. La pédagogie différenciée entre exigence d'égalité et droit à la différence. Suiza : Faculté de Psychologie et des Sciences de l'Éducation Université de Genève, 2005.

Quijano, Aníbal. Colonialidad del Poder, Eurocentrismo y América Latina. E. Lander (Ed.). Colonialidad Del Saber Y Eurocentrismo. Buenos Aires: UNESCOCLACSO, 2000.

Quilaqueo, Daniel. "Saberes educativos mapuche: racionalidad apoyada en la memoria social de los kimches". Revista Atenea, 505. 2012:79-102.

------ "Educación intercultural desde la Teoría del Control Cultural en contexto de diversidad sociocultural mapuche", en Cuadernos Interculturales 4. 2005:3750.

Quilaqueo, Daniel; Merino, María Eugenia. "Estereotipos y prejuicio étnico hacia los mapuches en textos complementarios a la asignatura de historia", en Revista de Educación Campo Abierto 23. 2003:119-135.

Quilaqueo, Daniel; Quintriqueo, Segundo. "Formación docente en educación intercultural para contexto mapuche en Chile", en Revista Cuadernos Interculturales 10. 2008:91-110.

------ "Saberes educativos mapuches: un análisis desde la perspectiva de los kimches", en Polis 9:26. 2010:56-69.

Quilaqueo, Daniel; Quintriqueo, Segundo.; Cárdenas, Prosperino. Educación, currículum e interculturalidad. Elementos sobre formación de profesores en contexto mapuche. Santiago de Chile: Frasis Editores, 2005.

Quilaqueo, Daniel; Merino, María Eugenia; Saiz, José. "Representación social mapuche e imaginario social no mapuche de la discriminación percibida", en Revista Atenea 496. 2007:81-103.

Quilaqueo, Daniel; Fernández, César; Quintriqueo, Segundo. Interculturalidad en contexto mapuche. Neuquén: Educo, 2010.

Quilaqueo, Daniel; Quintriqueo, Segundo; Llanquinao, Gabriel. La formation des maîtres en éducation interculturelle: les nouveaux contextes d'enseignement de la langue mapuzungun, en Abdallah El Mountassir et Louis-Jacques Dorais (Sous la direction), L'enseignement des langues vernaculaires: défis linguistiques, méthodologiques et socio-économiques. L'Harmattan. 2012:261289. 
Quintriqueo, Segundo. "Posibilidades y limitaciones de la educación intercultural en contexto mapuche", en Revista CUHSO 17:1. 2009:21-33.

------ Implicancias de un modelo curricular monocultural en contexto mapuche. Temuco Chile: Editorial LOM, 2010.

Quintriqueo, Segundo; Torres, Héctor; Gutiérrez, Maritza; Sáez, Dykssa. "Articulación entre el conocimiento cultural mapuche y el conocimiento escolar en ciencia", en Educación y Educadores 14:3. 2011:475-492.

Rockwell, Elsie. La escuela cotidiana. México: Fondo de Cultura Económica, 1995.

Saiz, José; Merino, María y Quilaqueo, Daniel. Meta estereotipos sobre los indígenas mapuches de Chile". Interdisciplinaria, 26: 1. 2009:23-48.

Sartorello, Stefano. "Una perspectiva crítica sobre interculturalidad y educación intercultural bilingüe: El caso de la Unión de Maestros de la Nueva Educación para México (UNEM) y educadores independientes en Chiapas", en Revista latinoamericana de educación inclusiva 3:2. 2009:77-90.

Schmelkes, Sylvia. Indígenas rurales, migrantes, urbanos: una educación equivocada, otra educación posible. A. Marchesi y M. Poggi (Coords.). Presente y futuro de la educación iberoamericana. Madrid: Egraf S.A., 2010.

Sichra, Inge; López, Luis Enrique. "La educación en áreas indígenas de América Latina", en Qinasay, Revisa de educación Intercultural Bilingüe 1;1 (2003):15-26.

Sleeter, Christine; Grant, Carl. Making choices for multicultural education: Five approaches to race, class, and gender. Columbus, $\mathrm{OH}$ : Merrill, 1988.

Tamagno, Liliana. Pueblos indígenas. Interculturalidad, colonialidad, política. Buenos Aires: Editorial Biblos, 2009.

Todorov, Tzvetan. Las morales de la historia. Barcelona: Ediciones Paidós Ibérica, S. A., 1993.

Torres, Héctor; Quilaqueo, Daniel. "Conceptos de tiempo y espacio entre los mapuches: racionalidad educativa", en Papeles de Trabajo. Cent. Estud. Interdiscip. Etnolingüíst. Antropol. Sociocult. 22 (2011):13-27.

Van Dijk, Teun. Racismo y discurso en América Latina. Barcelona: Editorial Gedisa, 2007.

Verma, Gattendra. Educación multicultural: problema de la investigación. T. Husén y S. Opper (Eds.). Educación multicultural y multilingüe. Madrid: Narcea, 1984.

Walsh, Catherine. "Interculturalidad, conocimientos y decolonialidad", en Signo y Pensamiento 24:46. 2005:39-50.

------ "Interculturalidad, colonialidad y educación", en Revista Educación y Pedagogía 19:48. 2007:25-35.

------- "Interculturalidad, plurinacionalidad y decolonialidad: las insurgencias políticoepistémicas de refundar el Estado", en Tabula Rasa 9. 2008:131-152.

Wieviorka, Michel. Une société fragmentée? Le multiculturalisme en débat. Paris: La Découverte, 1996. 